\title{
Performance of the CMS Electromagnetic Calorimeter in LHC Run II
}

\author{
N. Bartosik* for the CMS Collaboration \\ INFN Sezione di Torino, Turin, Italy \\ E-mail: nazar.bartosiketo.infn.it
}

\begin{abstract}
Many physics analyses using the Compact Muon Solenoid (CMS) detector at the LHC require accurate, high resolution electron and photon energy measurements, which are provided by the electromagnetic calorimeter (ECAL). During the Run II at the LHC it has been operating with proton-proton collisions at $13 \mathrm{TeV}$ center-of-mass energy, $25 \mathrm{~ns}$ bunch spacing, and an unprecedented instantaneous luminosity. High pileup levels (simultaneous collisions) and the ageing of crystals from exposure to large particle fluences necessitate a retuning of the ECAL readout, trigger thresholds, and reconstruction algorithms, to maintain the best possible performance in these increasingly challenging conditions. In addition, the energy response of the detector must be precisely calibrated and monitored using the expected response of the detector in physics events as well as using dedicated monitoring hardware. This work presents the new reconstruction algorithm and calibration strategies that have been implemented at CMS and the excellent performance achieved by the ECAL during Run II.
\end{abstract}

XXIX International Symposium on Lepton Photon Interactions at High Energies - LeptonPhoton2019 August 5-10, 2019

Toronto, Canada

${ }^{*}$ Speaker. 


\section{Introduction}

One of the primary goals of the Compact Muon Solenoid (CMS) experiment [1] was the observation of the Higgs boson and measurement of its properties. A crucial component of the CMS detector that makes this possible is the electromagnetic calorimeter (ECAL), which measures energies of electromagnetically interacting particles with high precision. Precise measurement of the diphoton invariant mass peak with resolution up to $1 \%$ requires precise energy reconstruction of each individual photon, as well as its position. The increased instantaneous luminosity delivered by the LHC during Run II poses additional challenges due to higher radiation levels and more energy deposits from concurrent (pileup) p-p collisions.

\section{Structure of the CMS ECAL}

The CMS ECAL is a compact, hermetic, homogeneous colorimeter with a fine spatial granularity, which is small enough to fit inside the superconducting solenoid, while providing excellent energy resolution. It consists of 75,848 $\mathrm{PbWO}_{4}$ scintillating crystals, arranged in a central barrel section (EB) and two endcaps (EE). Each crystal has a length of about 27 (26) radiation lengths in EB (EE) and lateral dimensions comparable to a Moliére radius $(2.19 \mathrm{~cm})$. In front of EE there is also a preshower detector (ES) with 3 radiation lengths of tungsten absorber followed by silicon strip sensors to discriminate between photons and $\pi^{0} \mathrm{~s}$.

The light from each crystal is read out by an avalanche photodiode (APD) in EB or a vacuum phototriode (VPT) in EE. The light yield of the crystals and the gain of the photodetectors strongly depend on temperature, therefore ECAL is operated at a very stable $T=18 \pm 0.05^{\circ} \mathrm{C}$, which is maintained by a dedicated water cooling system and temperature monitoring sensors.

\section{Energy reconstruction and calibration}

High-energy electromagnetic showers are typically spread over multiple crystals. Furthermore, the presence of dead material in front of the ECAL leads to electron bremsstrahlung and photon conversions that form additional energy deposits spread in $\phi$ by the strong magnetic field. In order to collect all this energy, superclusters of crystals are formed, starting from smaller clusters around local maxima of energy deposits, which are then dynamically combined with other clusters in $\phi$ to recover potentially radiated energy. The full energy of the shower is then reconstructed by summing signal amplitudes from each crystal in the supercluster and applying a number of corrections:

$$
E_{e / \gamma}=F_{e / \gamma} \cdot\left[G \cdot \sum_{i} S_{i}(t) C_{i} A_{i}\right]+E_{\mathrm{ES}} .
$$

In Eq. 3.1 the global $\mathrm{ADC}$ to $\mathrm{GeV}$ scale $(G)$ is applied to a sum of signal amplitudes $\left(A_{i}\right)$ from each crystal $i$ of the supercluster, corrected by intercalibration coefficients $\left(C_{i}\right)$, accounting for variations of individual crystal responses and readout efficiencies. A special multivariate correction $\left(F_{e / \gamma}\right)$ is applied to the total supercluster energy, which takes into account the particle type, geometry, and material effects [2]. The energy deposits in the preshower $\left(E_{\mathrm{ES}}\right)$ are also added for showers in the endcap region. The effect of these corrections is shown in Fig. 1. 

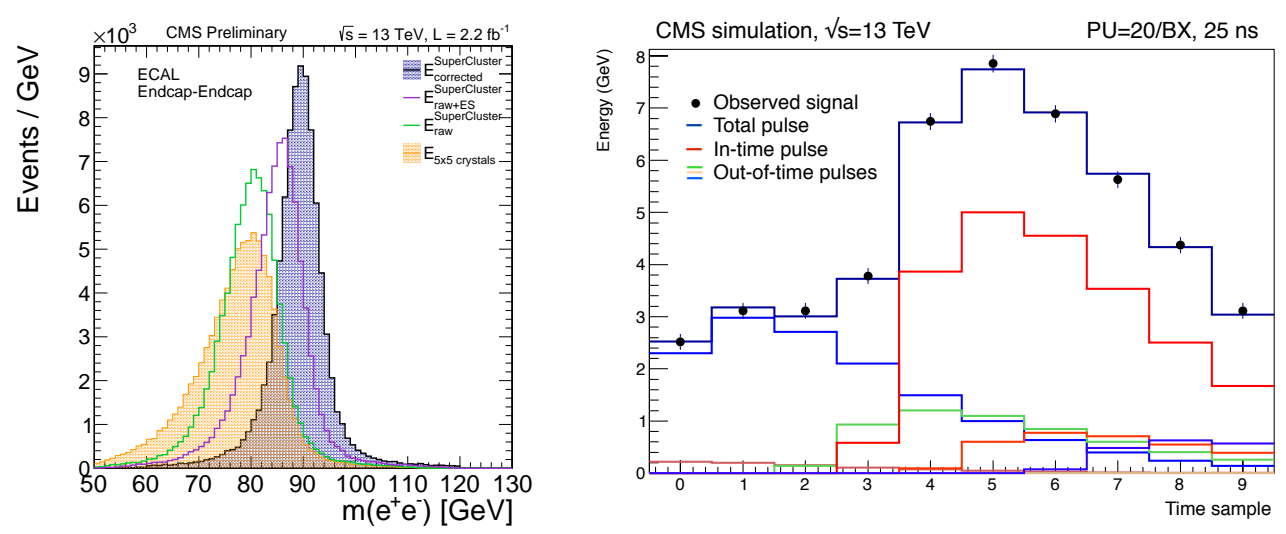

Figure 1: Left: Improvement to the $Z \rightarrow e^{+} e^{-}$energy scale and mass resolution from the application of super clustering and cluster corrections. Right: Example of fitted pulses for simulated events with 20 average pileup interactions and $25 \mathrm{~ns}$ bunch spacing, for a signal in the endcaps.

The signal amplitude $\left(A_{i}\right)$ from each crystal is corrected for potential pileup contributions before entering Eq. 3.1, making use of 10 consecutive samples of digitized APD/VPT pulse. While simply a weighted sum of the 10 samples was sufficient to calculate the pulse amplitude during Run I, a more sophisticated multifit approach is adopted for Run II [3]. Following this approach, a template fit of 1 in-time and up to 9 out-of-time pulses is performed to extract the desired pulse shape used for the amplitude calculation, as shown in Fig. 1.

\section{ECAL performance}
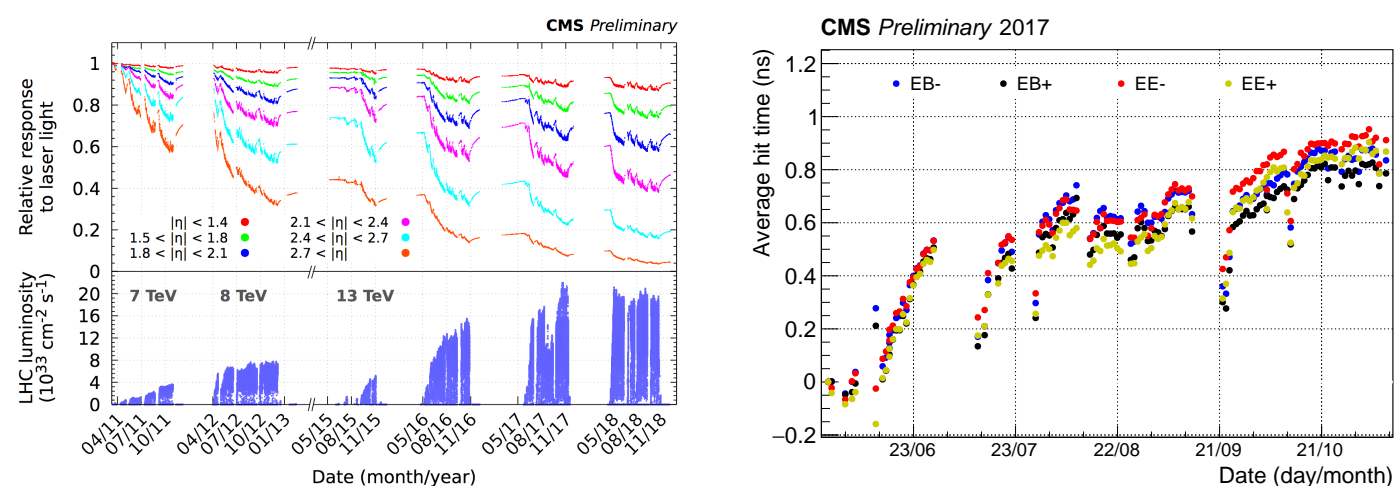

Figure 2: Left: Evolution of the relative response to the laser light injected in the ECAL crystals, averaged over all crystals in bins of pseudorapidity, for the data taking periods during 2011-2018. Right: Evolution of the average hit time for the positive and negative sides of EB and EE, as measured with 2017 data.

The light yield of the crystals is affected by the reduction of transparency under irradiation and its partial recovery through self-annealing in the absence of radiation, as shown in Fig. 2. The crystal transparency is continuously monitored with a dedicated laser system, and the corresponding corrections $\left(S_{i}(t)\right)$ are regularly updated to minimise time variations of crystal response. These variations also affect the pulse timing, which is crucial for robust pileup suppression. Timing shifts 
below 200 ps have a negligible effect on reconstruction, thus timing conditions are updated in steps of 200 ps, as shown in Fig. 2.

The single-electron energy resolution is derived from the invariant mass of $Z \rightarrow e^{+} e^{-}$candidates. This evaluation is done separately for electrons strongly affected by bremsstrahlung and for electrons with almost no bremsstrahlung, which are presented in Fig. 3. Resolution up to $1.6 \%$ is achieved in the central region and is better than $5 \%$ in the forwardmost region.

Dedicated alignment of the preshower detector by iteratively matching the ES hits to recontructed charged tracks significantly improves the reconstructed position, with the precision that is within the resolution of the silicon strips, as shown in Fig. 3.
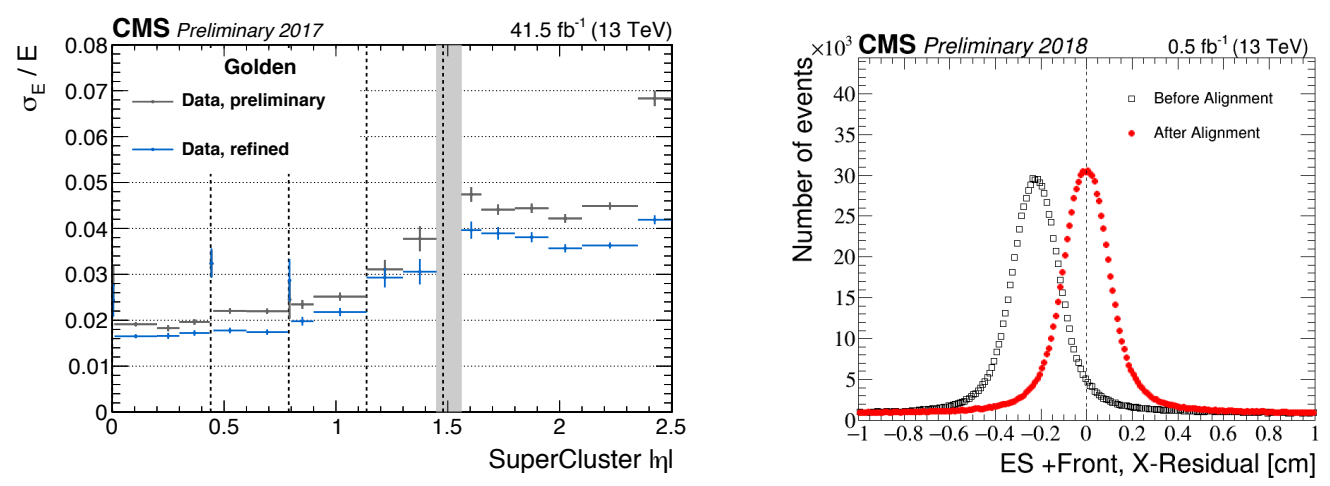

Figure 3: Left: Relative energy resolution for electrons with minimal effect of bremsstrahlung obtained with full 2017 calibration data (blue) or only correction for time-dependent effects (grey). Right: Preshower hit residuals in the local X coordinate before and after alignment, measured with 2018 data.

\section{Conclusion}

The CMS ECAL has been operating with excellent performance throughout LHC Run II, providing a wide range of physics analyses with precise measurements of particle energies. This level of performance was achieved thanks to continuous monitoring of detector conditions and regular updates of its calibration constants, as well as a number of improvements to the readout and reconstruction algorithms to cope with the increased pileup and radiation levels.

\section{References}

[1] CMS Collaboration, The CMS experiment at the CERN LHC, JINST, 3, S08004 (2008)

[2] CMS Collaboration, Energy calibration and resolution of the CMS electromagnetic calorimeter in $\mathrm{pp}$ collisions at $\sqrt{s}=7 \mathrm{TeV}$, JINST, 8, P09009 (2013)

[3] CMS Collaboration, Precision electromagnetic calorimetry at the energy frontier: CMS ECAL at LHC Run 2, arXiv:1510.02745 (2015) 